\title{
Bit-Interleaved Coded Modulation Systems with Iterative Decoding and Partial Reusing QAM Signal Points
}

\author{
Nguyen Quang Tuan ${ }^{1}$, Do Quoc Trinh ${ }^{2}$, Tran Xuan Nam ${ }^{2}$, Dinh The Cuong ${ }^{2}$ \\ ${ }^{1}$ Dept. of Communications, Ministry of Public Security \\ ${ }^{2}$ Le Qui Don Technical University, Hanoi, Vietnam
}

Correspondence: Dinh The Cuong, cuongdt@mta.edu.vn

Manuscript communication: received 7 July 2011, revised 25 October 2011

\begin{abstract}
A new method of reusing a subset of an M-QAM signal constellation is presented for Bit-Interleaved Coded Modulation systems with Iterative Decoding (BICM-ID). In addition to the remapping of high-energy signals to low-energy signals in order to save the average signal energy as a shaping technique known so far, a new scheme of remapping low-energy signals to high-energy signals is proposed in order to gain the equivalent distance when decoding bits, often called the Squared Euclidean Weights (SEW). Numerical analysis and simulation results show that the partial reuse of the signals in the BICM-ID system with 16-QAM can improve the system performance in terms of lowering the bit error rate in the error floor region.
\end{abstract}

Keywords- BICM-ID system, signal constellation shaping.

\section{INTRODUCTION}

In digital communication the transmission rate (bit/sec) is equal to the product of the symbol rate $r(\mathrm{sym} / \mathrm{sec}$ ) and the number $m$ (bit/sym) of bits carried by a modulation symbol transmitted over the channel. In this relationship the symbol rate represents the bandwidth resource of the communication channel and the modulation order $m$ represents its resource in the signal-to-noise power ratio (SNR). In the power-limited regime one can sacrifice the bandwidth to achieve the channel capacity by using powerful binary codes in combination with binary modulation like Binary Phase Shift Keying (BPSK). On the other hand, in the band-limited regime, when $r$ cannot be increased, the solution then is to combine binary coding with $M$ ary modulation, $M=2^{m}, m \geq 2$. Coded modulation (CM) schemes with high spectral efficiency include Block Coded Modulation (BCM) [1] and Trellis Coded Modulation (TCM) [2]. Many coded modulation schemes give large coding gains while having good algebraic structures which allow relatively simple encoding/decoding algorithms [3]. However, there are at least two points that limit the application of BCM and TCM. Namely, that both the two schemes are good for transmission over Additive White Gaussian Noise (AWGN) channels while performing badly in fading channels $[4,5]$. This leads to the need of powerful binary coding in order to compensate the increase in average signal energy which, in turn, is necessary for obtaining the required total gain. As an improvement in digital transmission over fading channels, the BitInterleaved Coded Modulation (BICM) [6] introduces time diversity by using bit interleaving between the encoding and modulation. In order to save the signal average energy while achieving high spectral efficiency, coded modulation schemes often use signal constellation shaping techniques, like trellis shaping and shell mapping [7]. As an alternative shaping technique, the use of partially overlapped signal sets has also been proposed in [8].

The application of Iterative Decoding (ID) principles to the BICM schemes by exchanging soft information between a Soft-Input Soft-Output (SISO) decoder and a Symbol-to-Bit Converter (SBC), working as a softoutput demodulator, has allowed the system to perform well on both Gaussian and fading channels [9]. In BICM-ID systems, the mapping from $m$-bit blocks to $M$-point signal constellation $\left(M=2^{m}, m \geq 2\right)$ plays an important role. While the Gray mapping is optimum for the systems without soft information feedback [6], Set Partitioning (SP) mapping, Anti-Gray mapping and other techniques give rise to better performance in the presence of feedback in BICM-ID systems. Nevertheless, like other systems employing ID, the Bit Error Rate (BER) curves of BICM-ID systems also have a water-fall part and an error floor part. Most research efforts so far have focused on methods for mapping onto multi-point signal constellations so that a) the water-fall happens earlier (at a smaller SNR) and b) the error floor happens at lower values of BER [10-12].

In this paper we study the signal constellation shaping in the BICM-ID scheme. Unlike techniques that use relatively complex shaping codes [13] or reuse the whole signal constellation [14], we develop the technique called partially overlapped signal subsets presented in [8] for TCM which reuses only a part of the signal constellation. Employing the feedback of the 


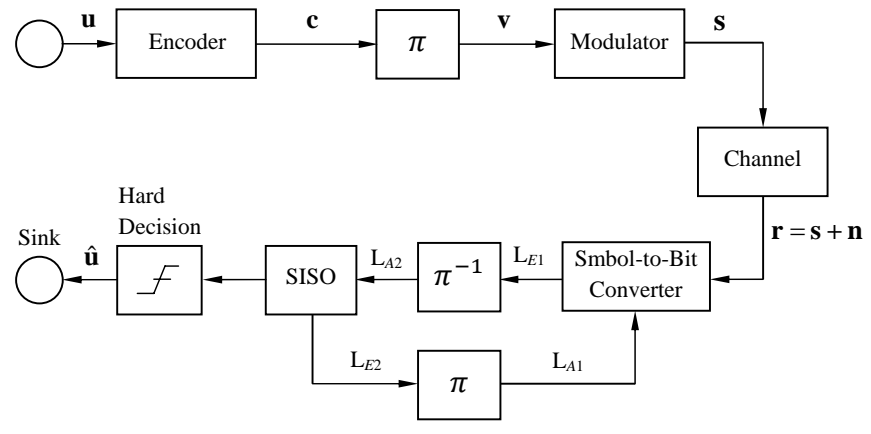

Figure 1. The block diagram of the BICM-ID system

soft information from the encoder to the demodulator, in addition to the reuse of low-energy signal points instead of high-energy signal points in order to reduce the average energy of signals transmitted over the channel we propose a new method for reusing of highenergy signal points instead of low-energy signal points in order to lower the BER value at the error floor region.

The paper is organized as follows. Section ?? describes the system model. The new proposal for the BICM-ID with 16-QAM is discussed in details in Section 3, together with simulation results in Section 4. Finally, conclusions are given in Section 5.

\section{The System Model}

Figure 1 shows the structure of the BICM-ID system with iterative decoding/demodulation at the receiver end. At the transmitter end an encoder output bit sequence $\mathbf{c}=\left(c_{1}, c_{2}, \ldots, c_{N}\right)$ is fed to the bit interleaver $\pi$ of length $N$, which performs index interleaving $\boldsymbol{v}_{i}=c_{\boldsymbol{\pi}(i)}=c_{j}$, where $\pi(i)=j, 1 \leq i, j \leq N$. For a given $M$-ary modulation scheme $\left(M=2^{m}, m \geq 2\right)$, consider a mapping rule $\mu:\left(v_{t 1}, v_{t 2}, \ldots, v_{t m}\right) \rightarrow s_{t}$, where $s_{t}$ is selected from the signal constellation $S$ and the time index $t$ runs from 1 to $N / m$. The interleaver length $N$ is chosen such that $N / m$ is an integer.

In this paper we consider Recursive Systematic Convolutional (RSC) encoders with a coding rate $R$. Each information sequence $\mathbf{u}$ of length $K=R N$ is encoded into the sequence c consisting of information bits and parity check bits. After being interleaved by $\pi$ the bits in the sequence $v$ are grouped into $m$-bit blocks, each is then mapped onto the constellation $S$ to form the signal sequence $\mathbf{s}=\left(s_{1}, s_{2}, \ldots, s_{N / m}\right)$, which is transmitted over the channel. We consider the transmission over an additive white Gaussian noise (AWGN) channel, for which the received signal vector can be expressed as $\mathbf{r}=\mathbf{s}+\mathbf{n}$, where $\mathbf{n}=\left(n_{1}, n_{2}, \ldots, n_{N / m}\right)$ is the noise vector and the noise samples $n_{t}$ are independent and identically distributed (i.i.d.), with zero mean, complexvalued and with noise variance $\sigma^{2}$ for both real and imaginary part. The SNR at the receiver is $E_{b} / N_{0}=$ $E_{s} /\left(N_{0} R m\right)$, where $E_{b} / N_{0}$ is the average transmitted energy per information bit, $E_{S}$ is the average transmitted energy per signal, and $N_{0}=2 \sigma^{2}$ is the one-side noise power spectral density.

The Symbol-to-Bit Converter takes in the channel output observation $\mathbf{r}$ and the a priori information $\mathbf{L}_{A 1}$ of $N$ coded bits and computes the extrinsic information $\mathbf{L}_{E 1}$, which in turn is de-interleaved and fed to the SISO decoder as the a priori information $\mathbf{L}_{A 2}$. The SISO decoder uses the Log-MAP algorithm in computation of the extrinsic information $\mathbf{L}_{E 2}$ for $N$ coded bits in the form of $N$ Log-Likelihood Ratio (LLR) values, which will be interleaved and used as the a priori information $\mathbf{L}_{A 1}$ in the next iteration.

Let $v$ be the interleaved version of the encoder's output $\mathbf{c}$ of $N$ coded bits, the LLR value for the bit $v_{t j}, 1 \leq t \leq N / m, 1 \leq j \leq m$ based on the channel observation $\mathbf{r}=\left(r_{1}, r_{2}, \ldots, r_{N / m}\right)$ is defined as

$$
L_{D}\left(v_{t j}\right)=\ln \frac{P\left(v_{t j}=1 \mid \mathbf{r}\right)}{P\left(v_{t j}=0 \mid \mathbf{r}\right)} .
$$

Although $\mathbf{c}$ has some structure inserted in by the RSC encoder, we can assume that bits in $v$ are independent due to the interleaving. Furthermore, the modulator is assumed to be memoryless, hence each bit group $v_{t}=$ $\left(v_{t 1}, v_{t 2}, \ldots, v_{t m}\right)$ depends only on the received signal $r_{t}$, $1 \leq t \leq N / m$. Then we have

$$
P\left[v_{t j}=u \mid r_{t}\right]=\frac{p\left(r_{t} \mid \boldsymbol{v}_{t}, v_{t j}=u\right) P\left[\boldsymbol{v}_{t}, v_{t j}=u\right]}{p\left(r_{t}\right)} .
$$

In (2), $p\left(r_{t} \mid \boldsymbol{v}_{t}, v_{t j}=u\right)$ is the probability density function of the received signal $r_{t}$ conditioned on the modulation bit block $v_{t}$ having $v_{t j}=u \in\{0,1\}$, and $P\left[\boldsymbol{v}_{t}, v_{t j}=u\right]$ is the a priori probability of $\boldsymbol{v}_{t}$ with $v_{t j}=u$. The unconditioned probability density function $p\left(r_{t}\right)$ is cancelled by division under the log-function in (1).

Referring to the presentation in [15] we can rewrite (1) as

$$
\begin{aligned}
L_{D}\left(v_{t j} \mid r_{t}\right)= & L_{A}\left(v_{t j}\right)+ \\
& \ln \frac{\sum_{\boldsymbol{v}_{t} \in v_{j,+1}} P\left(r_{t} \mid \boldsymbol{v}_{t}\right) \exp \left(\frac{1}{2} \boldsymbol{v}_{t,[j]}^{T} \mathbf{L}_{A,[j]}^{T}\right)}{\sum_{\boldsymbol{v}_{t} \in v_{j,-1}} P\left(r_{t} \mid \boldsymbol{v}_{t}\right) \exp \left(\frac{1}{2} \boldsymbol{v}_{t,[j]}^{T} \mathbf{L}_{A,[j]}^{T}\right)} .
\end{aligned}
$$

Here $v_{t,[j]}$ is formed from $v_{t}$ by deleting the $j$-th component, and $\mathbf{L}_{A,[j]}$ is obtained from the a priori probability vector of $\boldsymbol{v}_{t}$ by deleting the $j$-th component. Then $\mathbf{L}_{D}$ is the sum of the a priori information $\mathbf{L}_{A}$ and the extrinsic information $\mathbf{L}_{E}$. The decoder and the demodulator exchange the extrinsic information $\mathbf{L}_{E}$ :

$$
L_{E}\left(v_{t j} \mid r_{t}\right)=\ln \frac{\sum_{v_{t} \in v_{j,+1}} P\left(r_{t} \mid \boldsymbol{v}_{t}\right) \exp \left(\frac{1}{2} \boldsymbol{v}_{t,[j]}^{T} \mathbf{L}_{A,[j]}^{T}\right)}{\sum_{v_{t} \in v_{j,-1}} P\left(r_{t} \mid \boldsymbol{v}_{t}\right) \exp \left(\frac{1}{2} \boldsymbol{v}_{t,[j]}^{T} \mathbf{L}_{A,[j]}^{T}\right)} .
$$

For the AWGN channel we have, with $s_{t}=\mu\left(\boldsymbol{v}_{t}\right)$,

$$
P\left(r_{t} \mid \boldsymbol{v}_{t}\right)=\frac{\exp \left(-\frac{1}{2}\left\|r_{t}-s_{t}\right\|^{2}\right)}{\sqrt{2 \pi \sigma^{2}}} .
$$

Based on the observation of (4) and (5) we have two following remarks.

Remark 1: Let us assume the perfect feedback of extrinsic information from the SISO decoder for the RSC code to the Symbol-to-Bit Converter which uses (4) and (5) to compute its output extrinsic information 
$L_{E}\left(v_{t j} \mid r_{t}\right)$ with the exact knowledge of $\mathbf{L}_{A,[j]}$. In other words, in (4) we have in the enumerator the summation only over one signal $s_{t,+1}$ with the label $v_{t i}$, $1 \leq i \leq m, i \neq j, v_{t j}=1$ and in the dominator the summation only over one signal $s_{t,-1}$ with the label $v_{t i}, 1 \leq i \leq m, i \neq j, v_{t j}=0$. In combination of (5), we have the extrinsic information $L_{E}\left(v_{t j} \mid r_{t}\right)$ then depends only on the observed signal $r_{t}$ and the Squared Euclidean Distance of the pair of signal points whose binary labels differ only in the $j$-th position. Namely, $L_{E}\left(v_{t j} \mid r_{t}\right)=\left(\left\|r_{t}-x_{t,-1}\right\|^{2}-\left\|r_{t}-x_{t,+1}\right\|^{2}\right) / 2 \sigma^{2}$.

Remark 2: Suppose that a signal point $\mathbf{s}_{t}$ has two different binary labels $v_{t}$ and $v^{\prime}{ }_{t}$. According to (5) we have $P\left(r_{t} \mid \boldsymbol{v}_{t}\right)=P\left(r_{t} \mid \boldsymbol{v}_{t}{ }_{t}\right)$. Due to the feedback from the SISO decoder in the form of a priori probabilities $L_{A,[j]}$ and the exponents $\exp \left(\frac{1}{2} \boldsymbol{v}_{t,[j]}^{T} \mathbf{L}_{A,[j]}^{T}\right) \neq \exp \left(\frac{1}{2} \boldsymbol{v}_{t,[j]}^{\prime T} \mathbf{L}_{A,[j]}^{T}\right)$, we still can obtain $L_{E}\left(v_{t j} \mid r_{t}\right) \neq L_{E}\left(v_{t j}^{\prime} \mid r_{t}\right)$. This means some signal points may be used to transmit more than one binary labels.

The serial concatenation structure of the encoder/modulator pair at the transmitter and of the demodulator/decoder pair at the receiver in the BICM-ID scheme seems to be simpler than the parallel concatenation structure of turbo encoders. The bit interleaving in the BICM scheme gives rise to the large temporal diversity to help the system robustness in the fading channel. Due to the iterative decoding where the a priori information of $m-1$ bits per received symbol is used to improve the detection of the remained bit, the $M$-ary modulation can be considered as a set of $2^{m-1}$ independent BPSK modulators which might allow mappings that, in turn, give rise to good performance of the BICM-ID system in the AWGN channel.

\section{Partial Reusing of 16-QAM Signal POINTS}

Consider an $M$-QAM constellation with $M=2^{m}$ signal points each of which is given by a complex number $s_{k}=a_{k}+i b_{k}, 1 \leq k \leq M$, where $i=\sqrt{-1}$ and $a_{k}, b_{k}=$ $\pm 1, \pm 3, \ldots, \pm 2^{m}-1$. When the signal points are used equally-likely we have

$$
E_{s}=\frac{1}{M} \sum_{k=1}^{M}\left(a_{k}^{2}+b_{k}^{2}\right) .
$$

The left hand side of Figure 2 shows the configuration of the 16-QAM constellation. In this paper we consider the optimum mapping of the 16-QAM constellation [10]. This mapping has been stated to be optimum in the sense that it has an optimum distance spectrum which allows to reach low error rates. For convenience, let us enumerate from 1 to 16 for signal points $s_{k}, 1 \leq$ $k \leq 16$, from left to right and from upper rows to lower rows. The optimum mapping is now represented as $\mu_{\mathrm{opt}}=[13,6,7,16,3,12,14,5,8,15,9,2,10,1,4,11]$. Each of the numbers $1 \leq k \leq 16$ in the previous vector denotes the signal point $s_{k}$ whose binary label has a decimal value of $(k-1)$. The binary labeling of the 16QAM points in the optimum mapping is presented in

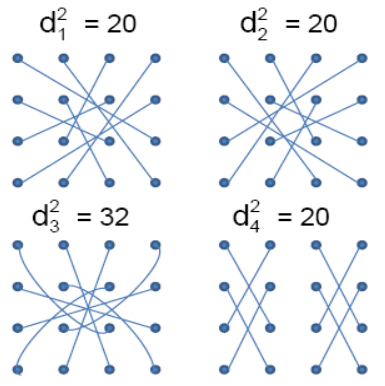

Figure 2. The optimum mapping of the 16-QAM points

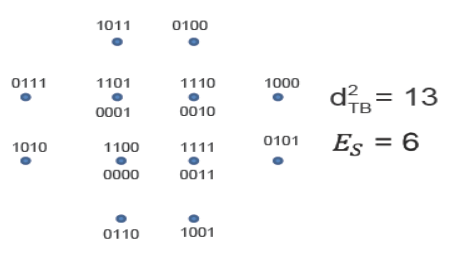

(a)

$$
\begin{aligned}
& \begin{array}{ll}
1101 & 1110 \\
1011 & \\
0100
\end{array} \\
& \stackrel{\circ}{0111} \quad \stackrel{\circ}{0001} \quad \stackrel{\circ}{0010} \quad \stackrel{\circ}{1000} \mathrm{~d}_{\mathrm{TB}}^{2}=19 \\
& 1010 \quad 1100 \quad 1111 \quad 0101 \quad E_{S}=8 \\
& \begin{array}{ll}
0110 & 1001 \\
010 & 01 \\
0000 & 0011
\end{array}
\end{aligned}
$$
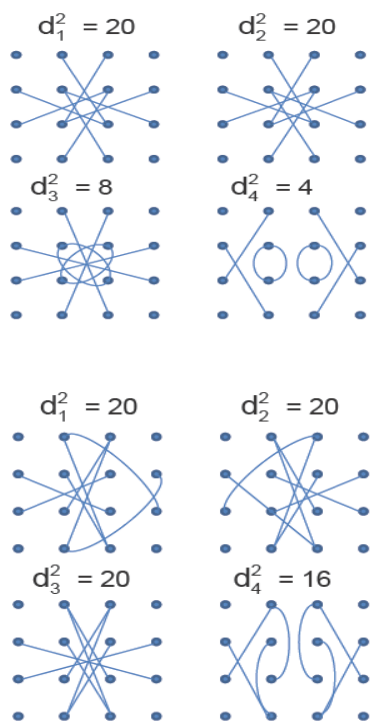

(b)
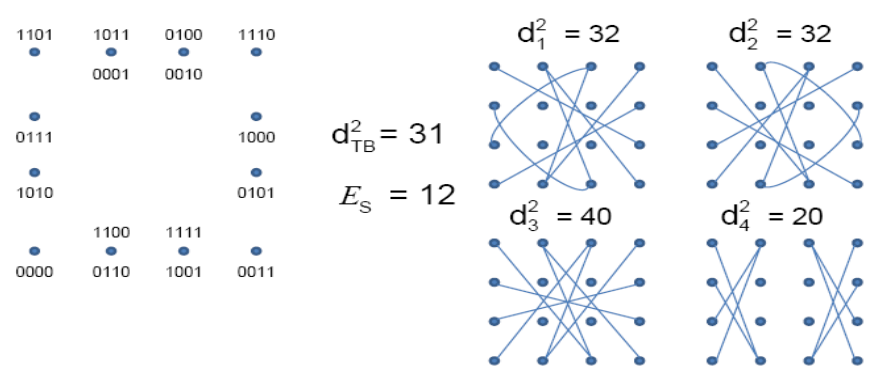

(c)
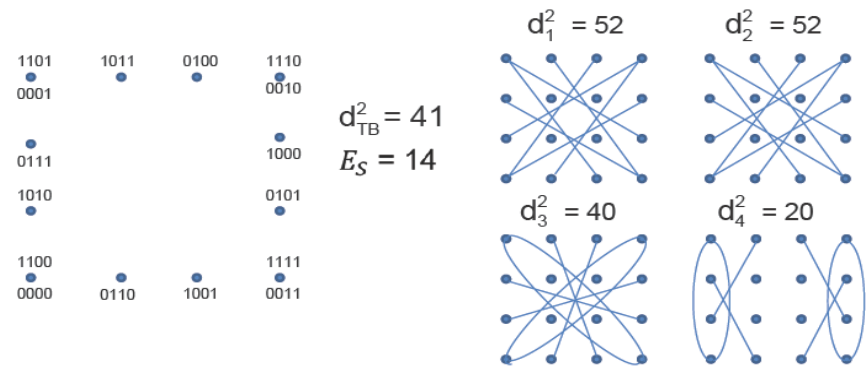

(d)

Figure 3. Different ways of reusing the 16-QAM points

Figure 2. The average energy of the 16-QAM constellation is equal to 10 .

We use Figure 3 to describe different ways of reusing a part of signal points in the 16-QAM constellation. Each of signal constellations in Figure 3 has only 12 signal points, meaning that four signal points are used twice frequently for transmission of 2 bit/sym in com- 
bination with rate-1/2 RSC codes. Each of reused signal points has then two binary labels, one of the signal point itself and the other is the label of the point that is not used for transmission. In Figure 3(a), four lowestenergy signals (points 6, 7, 9, and 10 of energy equal to 2) are reused for transmission instead of four highestenergy signals (points $1,4,13$, and 16 of energy equal to 18 ). The average energy of actually transmitted 16QAM signals now is equal to 6. Figure 3(b) presents the way of reusing secondary low-energy signals (points 2 , 3,14 , and 15 of energy equal to 10) for transmission instead of highest-energy signals (points 1, 4, 13, and 16 of energy equal to 18). The average energy of actually transmitted 16-QAM signals in this case is equal to 8 . While saving the average signal energy, we can see later in performance analysis that these ways of reusing lowenergy signal points cannot gain much in the average SED.

Contrarily, the reusing of high-energy points instead of low-energy points allows an increase of the average SED while sacrificing the average signal energy. In Figure 3(c), four secondary low-energy signals (points $2,3,14$, and 15 of energy equal to 10) are reused for transmission instead of four lowest-energy signals (points 6, 7, 9, and 10 of energy equal to 2). The average energy of actually transmitted 16-QAM signals now is equal to 12. Finally, Figure 3(d) presents the way of reusing highest-energy signals (points 1, 4, 13, and 16 of energy equal to 18) for transmission instead of lowestenergy signals (points $6,7,9$, and 10 of energy equal to 2 ). The average energy of actually transmitted 16-QAM signals in this case is equal to 14 .

To describe different ways of reusing 16-QAM signal points, we use the representation of a mapping rule as it has been presented in Section 3. We combine the notation of the optimum mapping $\mu_{\mathrm{opt}}=[13,6,7,16,3,12,14,5,8,15,9,2,10,1,4,11]$ and the rule of reusing to represent the way of reusing as a new mapping rule. For example, the way of reusing presented in Figure 3(a) can be now represented as a mapping rule $\mu_{\mathrm{opt}}=$ $[10,6,7,11,3,12,14,5,8,15,9,2,10,6,7,11]$, since point 10 is reused for point 13 , point 11 is reused for point 16 , point 6 is reused for point 1 , and point 7 is reused for point 4 . Similarly we have different mapping rules representing different ways of reusing 16-QAM signal points as they are given in Table I.

\section{Performance Analysis}

In general, let us consider the $j$-th bit in $m$-bit binary label of a signal point. Under the condition that the number of signal points $M$ satisfies $M=2^{m}$, for each signal point $s_{k}$ there is a signal point $s_{k *}$ whose $m$-bit label differs from the label of $s_{k}$ only in the $j$-th bit. Denote by $d_{j}^{2}\left(s_{k}\right)=\left\|s_{k}-s_{k *}\right\|^{2}$ the squared Euclidean distance (SED) between $s_{k}$ and $s_{k *}$. Given a mapping $\mu$ onto the M-QAM constellation, the SED $d_{j}^{2}\left(s_{k}\right)$ depends on the signal point $s_{k}$ and the bit position $j$. If this SED is constant for all signal points $s_{k}$ for each $j$, then the mapping is defined as to have a uniform error probability, that is the bit error probability in each position does not depend on the transmitted signal. In this case we have $d_{j}^{2}=d_{j}^{2}\left(s_{k}\right)$ independently of $s_{k}$. For other kinds of mappings, in this paper we propose to set

$$
d_{j}^{2}=\min _{1 \leq k \leq M} d_{j}^{2}\left(s_{k}\right),
$$

meaning that for the bound in (12) to take place we consider for each bit position the worst equivalent binary channel before averaging by (9) over $m$ bit in the binary label of the M-QAM point.

For $M=16$ and a mapping $\mu$ onto the 16-QAM signal constellation, let us define the SED Profile of $\mu$, denoted by $\operatorname{DP}(\mu)$, as an ordered set $\operatorname{DP}(\mu)=$ $\left(d_{1}^{2}, d_{2}^{2}, d_{3}^{2}, d_{4}^{2}\right)$ of SEDs under the mapping $\mu$. For example, Figure 2 shows the binary labeling of the 16QAM points in the optimum mapping, together with the average signal energy (6), the average SED (9), and the SEDs of each bit position (7). We have $\mathrm{DP}\left(\mu_{\mathrm{opt}}\right)=$ $(20,20,32,20)$ and $d_{T B}^{2}=23$, while $E_{s}=10$. In Figures 2 and 3, each pair of points linked by a line is used to define the SED $d_{j}^{2}\left(s_{k}\right)=\left\|s_{k}-s_{k *}\right\|^{2}$, for $1 \leq j \leq 4$ and $1 \leq k \leq 16$.

Developments on BICM-ID [9, 10, 12] have shown that, when the SNR is large enough (when the feedback is perfect), we can assume that each encoder output bit selects for transmission a binary channel of the SED $d_{j}^{2}, 1 \leq j \leq m$, with probability $1 / m$. Consequently coded bits are assumed to be transmitted over an equivalent BPSK modulation channel with an average signal energy (and also bit energy) equal to

$$
E_{b}^{*}=\frac{1}{4 m} \sum_{j=1}^{m} d_{j}^{2}=\frac{d_{\mathrm{TB}}^{2}}{4},
$$

where the average SED is computed as

$$
d_{\mathrm{TB}}^{2}=\frac{1}{m} \sum_{j=1}^{m} d_{j}^{2} .
$$

For a given $S N R=E_{b} / N_{0}$, the one-side power spectral density of the AWGN is

$$
N_{0}=\frac{E_{s}}{\frac{E_{b}}{N_{0}} R m},
$$

where $R$ is the coding rate of the convolutional encoder. Then the SNR of the equivalent BPSK channel is

$$
\frac{E_{b}^{*}}{N_{0}}=\left(\frac{1}{4 E_{s}} \sum_{j=1}^{m} d_{j}^{2}\right) \frac{E_{b}}{N_{0}} R=\frac{m R}{4} \frac{d_{T B}^{2}}{E_{s}} \frac{E_{b}}{N_{0}}
$$

It is well known that the bit error probability of a rate$b / c$ convolutional encoder over an AWGN channel with BPSK modulation and with the signal-to-noise ratio $E_{b}^{*} / N_{0}$ is upper bounded as [16]

$$
P_{b}<\left.\frac{1}{b} \frac{\partial T(D, I, L)}{\partial I}\right|_{I=1, L=1, D=\exp \left(-E_{b}^{*} / N_{0}\right)}
$$

where $T(D, I, L)$ is the extended transfer function of the error state diagram of the encoder. Thus we can 
Table I

Parameters of Different Ways of Partial Reusing 16-QAM Signals

\begin{tabular}{|l|l|l|l|l|l|}
\hline$p$ & Mapping rules & $E_{s}$ & $d_{T B}^{2}$ & $G_{p}$ & Description \\
\hline 1 & $\mu_{a}=[10,6,7,11,3,12,14,5,8,15,9,2,10,6,7,11]$ & 6 & 13 & 1.083 & Reuse 6, 7, 10, 11 instead of 1,4, 13,16 \\
\hline 2 & $\mu_{\mathrm{opt}}=[14,6,7,15,3,12,14,5,8,15,9,2,10,2,3,11]$ & 8 & 19 & 1.187 & Reuse 2, 3, 14, 15 instead of 1,4,13,16 \\
\hline 3 & $\mu_{b}=[13,6,7,16,3,12,14,5,8,15,9,2,10,1,4,11]$ & 10 & 23 & 1.150 & The original optimum mapping \\
\hline 4 & $\mu_{c}=[13,2,3,16,3,12,14,5,8,15,9,2,14,1,4,15]$ & 12 & 31 & 1.291 & Reuse 2, 3, 14, 15 instead of 6,7, 10,11 \\
\hline 5 & $\mu_{d}=[13,1,2,16,3,12,14,5,8,15,9,2,13,1,4,16]$ & 14 & 41 & 1.463 & Reuse 1, 4, 13,16 instead of 6, 7, 10,11 \\
\hline
\end{tabular}

use the bound (12) in conjunction with the equivalent SNR (11) to compare different ways of reusing 16-QAM signal points, provided that the same convolutional code is used in the BICM-ID system. Additionally, the form of (11) suggests that by taking the bit error probability of the same convolutional encoder at $E_{b} / N_{0}$ as a baseline for comparisons, the performance of the BICM-ID system with different ways of partial reuses depends on the reusing gain

$$
G=\frac{m R}{4} \frac{d_{\mathrm{TB}}^{2}}{E_{s}} .
$$

Namely, the system with a larger $G$ will have a smaller value of BER at the error floor region. In particular, the BICM-ID system using a rate- $1 / 2$ convolutional code in combination with 16-QAM has $G=\left(d_{T B}^{2} / E_{s}\right) / 2$, computed from (13) for $R=1 / 2$ and $m=4$. If the whole $M$-QAM signal constellation with $M=2^{m}$ is reused in transmission of $(m+1)$ bit/sym as it has been proposed in [14] the decrease of $d_{\mathrm{TB}}^{2}$ due to the assignment of the smaller SED in the distance profile to the $(m+1)$-th bit cannot be compensated with the increase of the number of bit/sym from $m$ to $(m+1)$, especially when $m$ is large. It is this fact itself has suggested a partial reuse of signal points in order to increase $G$.

Let $G_{p}$ denote the gain of the $p$-th mapping as shown in Table I, computed by using (13). Arranging in descending order of mapping gains, we have $G_{5}>$ $G_{4}>G_{3}>G_{2}>G_{1}$. This order means that, in the error floor region, the system without reusing signal points (the original 16-QAM constellation with $E_{s}=10$ ) outperforms the system that reuses lowest-energy points instead of highest-energy points $\left(E_{s}=6\right)$, however it is outperformed by three other ways of partial reusing 16-QAM signal points. This fact is confirmed by simulation results presented as BER curves versus $E_{b} / N_{0}$ $(\mathrm{dB})$, as shown in Figure 4. In this simulation the RSC encoder has the generators $[1,5 / 7]$, and the length of the random bit interleaver is equal to 4800 . Furthermore, Figure 4 also presents the upper bounds (12) for different ways of partial reusing $16-\mathrm{QAM}$ signal points, differentiated by average signal energy $E_{s}$. We note that the upper bounds are close to the simulation results in the error floor region and they reflect the similar relations as in the comparison by using reusing gain $G$. This supports the conclusion that the mappings $\mu_{b}$, $\mu_{c}$ and $\mu_{d}$ representing the ways of partial reusing 16QAM signal points under the optimum mapping in Figures 3(b), 3(c) and 3(d), respectively, allow to lower

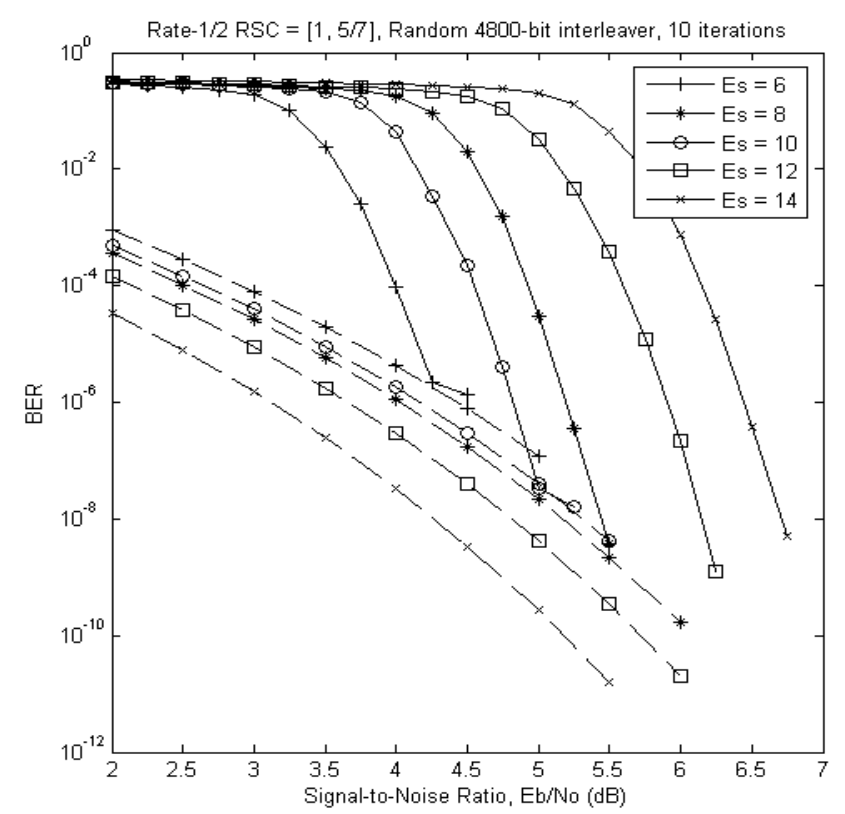

Figure 4. Simulation results (solid lines) of BICM-ID with partial reuse of 16-QAM signals and upper bounds (dash lines) using optimum mapping of [10].

the BER in the error floor region compared with the case when there is not any reuse.

Figure 5 shows the advantage of inline interleaving over overall interleaving. The simulation has been done with 4-state rate-1/2 RSC code of generators [1,5/7]. The interleaver's length was 10000 bits. Again the optimum mapping rule of [10] was applied to the 16-QAM constellation together with different ways of reusing signal points as depicted in Figure 3. It can be seen that BER curves perform the same relation when comparing different ways of reusing signal points. In addition, we note that the system with inline interleaving performs out the system with overall interleaving.

For comparison, we present in Figure 6 simulation results of different ways of reusing the 16-QAM signal points in the case of Modified Set Partitioning (MSP) mapping of [17]. Except the case of reusing lowest energy signal points instead of highest energy signal points (i.e. $E_{s}=6$ ), we observe the similar relations as it is noted with Figure 4. It seems that for each known mapping rule of the 16-QAM signal constellation there are ways of reusing its points such that the BER in the error floor region can be lowered. 


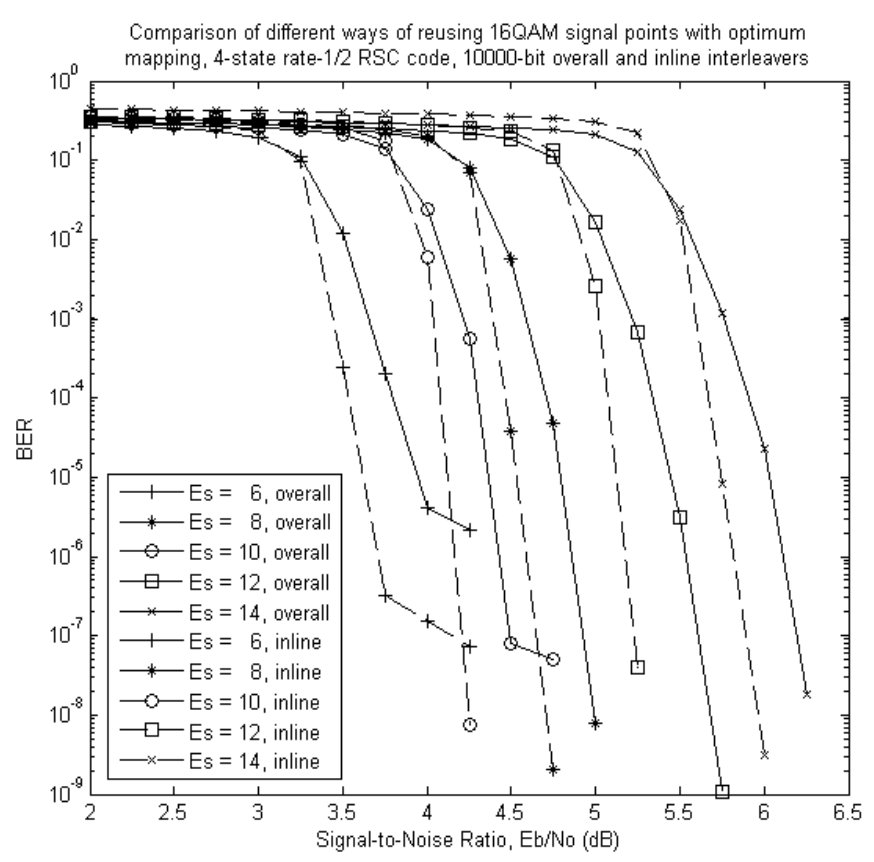

Figure 5. Performance of the BICM-ID system with different way of reusing 16-QAM signal points for overall and inline interleaving. 4-state rate-1/2 RSC code, 10 iterations.

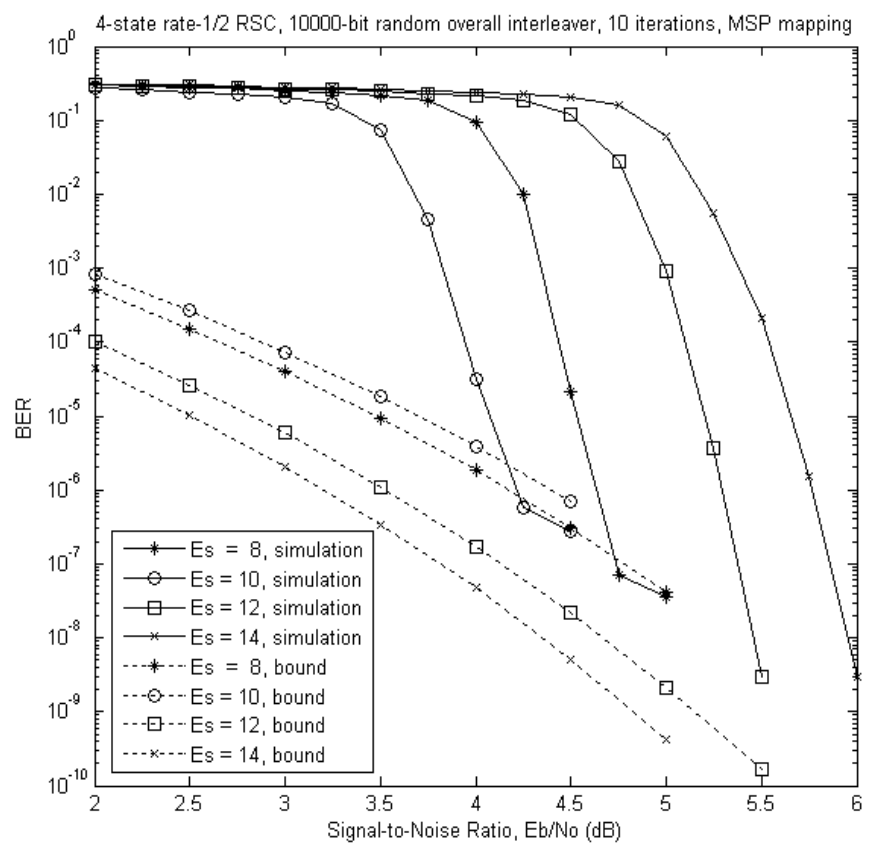

Figure 6. Simulation results (solid lines) of BICM-ID with partial reuse of 16-QAM signals and upper bounds (dash lines) using modified SP mapping of [17].

\section{Conclusion}

Thus, given a mapping rule, like the optimum mapping, of the 16-QAM constellation, it is simple to define the way of partial reusing signal points by redefining the given mapping rule. The simple analytical upper bound on the BER based on the encoder transfer function is possible due to the consideration that, for each given bits position in the signal point's binary label, the minimum SED dominates the BER property of the equivalent binary channel defined by this bit position. Two simple ways of comparison, the one by using analytical upper bound on BER and the other by defining the so called reusing gain, have shown that the technique of partial reuse of the 16-QAM signal points allows to improve the BER performance of the BICM-ID system in the error floor region, compared with the case of no reuse. This fact is confirmed by simulation results for the BICM-ID system using the optimum mapping of 16-QAM constellation and transmitting $2 \mathrm{bit} / \mathrm{sym}$. Finally we note that the existence of different ways of partial reusing signal points for the same signal constellation with different BER performance can be useful for systems where adaptive schemes are considered.

\section{REFERENCES}

[1] H. Imai and S. Hirakawa, "A new multilevel coding method using error-correcting codes," IEEE Transactions on Information Theory, vol. 23, no. 3, pp. 371-377, May 1977.

[2] G. Ungerboeck, "Channel coding with multilevel/phase signals," IEEE Transactions on Information Theory, vol. 28, no. 1, pp. 55-67, Jan. 1982.

[3] J. Forney, G. D., "Geometrically uniform codes," IEEE Transactions on Information Theory, vol. 37, no. 5, pp. 12411260, Sep. 1991.

[4] C. Schlegel and J. Costello, D. J., "Bandwidth efficient coding for fading channels: code construction and performance analysis," IEEE Journal on Selected Areas in Communications, vol. 7, no. 9, pp. 1356-1368, Dec. 1989.

[5] E. Zehavi, "8-PSK trellis codes for a Rayleigh channel," IEEE Transactions on Communications, vol. 40, no. 5, pp. 873-884, May 1992.

[6] G. Caire, G. Taricco, and E. Biglieri, "Bit-interleaved coded modulation," IEEE Transactions on Information Theory, vol. 44, no. 3, pp. 927-946, May 1998.

[7] U. Wachsmann, R. F. H. Fischer, and J. B. Huber, "Multilevel codes: Theoretical concepts and practical design rules," IEEE Transactions on Information Theory, vol. 45, no. 5, pp. 1361-1391, July 1999.

[8] V. K. Tran, T. C. Dinh, and T. Hashimoto, "Trellis coded modulation with partially overlapped signal subsets," in Proc. Int. Conf. Advanced Technologies for Communications (ATC 2008), Hanoi, Vietnam, 6-9 Oct. 2008, pp. 177-180.

[9] X. Li, A. Chindapol, and J. A. Ritcey, "Bit-interleaved coded modulation with iterative decoding and 8 PSK signaling," IEEE Transactions on Communications, vol. 50, no. 3, pp. 1250-1257, Aug. 2002.

[10] F. Schreckenbach, N. Gortz, J. Hagenauer, and G. Bauch, "Optimization of symbol mappings for bit-interleaved coded modulation with iterative decoding," IEEE Communications Letters, vol. 7, no. 12, pp. 593-595, Dec. 2003.

[11] F. Shreckenbach and G.Bauch, "Irregular signal constellations, mappings and precoder," in International Symposium on Information Theory and its Applications (ISITA 2004), Parma, Italy, 10-13 Oct. 2004.

[12] L. Szczecinski, H. Chafnaji, and C. Hermosilla, "Modulation doping for iterative demapping of bit-interleaved coded modulation," IEEE Communications Letters, vol. 9, no. 12, pp. 1031-1033, 2005.

[13] B. K. Khoo, S. Y. Le Goff, B. S. Sharif, and C. C. Tsimenidis, "Bit-interleaved coded modulation with iterative decoding using constellation shaping," IEEE Transactions on Communications, vol. 54, no. 9, pp. 1517-1520, Sep. 2006.

[14] P. Henkel, "Extended mappings for bit-interleaved coded modulation," in Proc. 17th IEEE Int. Symposium on Personal, Indoor and Mobile Radio Communications (PIMRC), Helsinki, 11-14 Sep. 2006, pp. 1-4. 
[15] B. M. Hochwald and S. ten Brink, "Achieving nearcapacity on a multiple-antenna channel," IEEE Transactions on Communications, vol. 51, no. 3, pp. 389-399, 2003.

[16] A. J. Viterbi and J. K. Omura, Principles of Digital Communication and Coding. McGraw-Hill, 1979.

[17] J. Tan and G. L. Stuber, "Analysis and design of symbol mappers for iteratively decoded BICM," IEEE Transactions on Wireless Communications, vol. 4, no. 2, pp. 662672, Mar. 2005

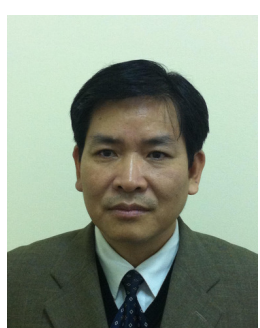

Nguyen Quang Tuan was born in 1964 in Hanoi. He received his B.Eng. at the University of Communication Technology in 1995, and M.Eng. at Hanoi University of Science and Technology in 1996. He works as a researcher at Department of Communications, Vietnam Ministry of Public Security. He is currently pursuing a PhD study at Le Qui Don Technical University.

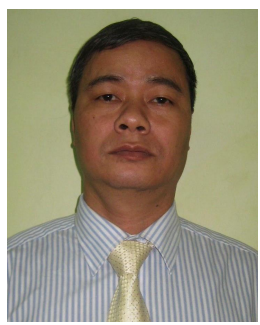

Do Quoc Trinh was born in 1961. He received his B.Eng. and Ph.D. degrees at Le Qui Don Technical University in 1982 and 2003, respectively. Dr. Do Quoc Trinh is currently a lecturer of Faculty of Radio-Electronics Engineering, Le Qui Don Technical University, Hanoi, Vietnam. His research interests are in areas of mobile communication and channel coding.

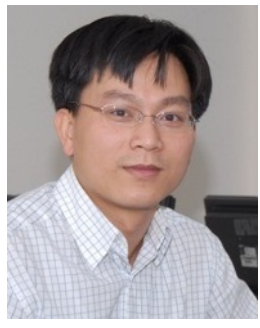

Xuan Nam Tran is currently an associate professor at Department of Communications Engineering at Le Quy Don Technical University Vietnam. He received his master of engineering (ME) in telecommunications engineering from University of Technology Sydney, Australia in 1998, and doctor of engineering in electronic engineering from The University of Electro-Communications, Japan in 2003. From November 2003 to March 2006 he was a research associate at the Information and Communication Systems Group, Department of Information and Communication Engineering, The University of Electro-Communications, Tokyo, Japan.

Dr. Tran research interests are in the areas of adaptive antennas, space-time processing, space-time coding and MIMO systems. He is the author and co-author of more than thirty technical papers published in international journals and conference proceedings. Dr. Tran is a recipient of the 2003 IEEE AP-S Japan Chapter Young Engineer Award. He is a member of IEEE, IEICE, and the RadioElectronics Association of Vietnam.

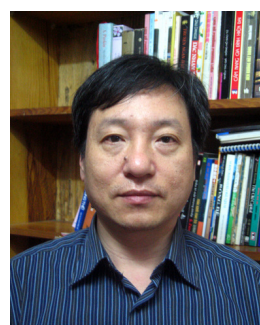

The Cuong Dinh was born in Hanoi, Vietnam, in 1963. He received the B.Eng. from Hanoi University of Technology in 1986, the M.Eng. and Dr.Eng. from the University of ElectroCommunication, Tokyo, Japan, respectively, in 1996 and 1999. He is an Associated Professor in Le Quy Don Technical University, Hanoi, Vietnam. His main interests are channel coding theory and its applications. 